\title{
Drug seller adherence to clinical protocols with integrated management of malaria, pneumonia and diarrhoea at drug shops in Uganda
}

Phyllis Awor ${ }^{1,2^{*}}$, Henry Wamani ${ }^{2}$, Thorkild Tylleskar ${ }^{1}$ and Stefan Peterson ${ }^{2,3,4}$

\begin{abstract}
Background: Drug shops are usually the first source of care for febrile children in Uganda although the quality of care they provide is known to be poor. Within a larger quasi-experimental study introducing the WHO/UNICEF recommended integrated community case management (iCCM) of malaria, pneumonia and diarrhoea intervention for community health workers in registered drug shops, the level of adherence to clinical protocols by drug sellers was determined.
\end{abstract}

Methods: All drug shops $(N=44)$ in the intervention area were included and all child visits $(N=7,667)$ from October 2011-June 2012 to the participating drug shops were analysed. Drug shops maintained a standard iCCM register where they recorded the children seen, their symptoms, diagnostic test performed, treatments given and actions taken. The proportion of children correctly assessed and treated was determined from the registers.

Results: Malaria management: 6,140 of 7,667 (80.1\%) total visits to drug shops were of children with fever. 5986 (97.5\%) children with fever received a malaria rapid diagnostic test (RDT) and the RDT positivity rate was 78\% (95\% Cl 77-79). 4,961/5,307 (93.4\%) children with a positive RDT received artemisinin combination therapy. Pneumonia management: after respiratory rate assessment of children with cough and fast/difficult breathing, 3,437 (44.8\%) were categorized as "pneumonia", 3,126 (91.0\%) of whom received the recommended drug-amoxicillin. Diarrhoea management: 2,335 (30.5\%) child visits were for diarrhoea with 2,068 (88.6\%) correctly treated with oral rehydration salts and zinc sulphate. Dual/Triple classification: 2,387 (31.1\%) children had both malaria and pneumonia and 664 (8.7\%) were classified as having three illnesses. Over $90 \%$ of the children with dual or triple classification were treated appropriately. Meanwhile, 381 children were categorized as severely sick (with a danger sign) with 309 (81.1\%) of them referred for appropriate management.

Conclusion: With the introduction of the iCCM intervention at drug shops in Eastern Uganda, it was possible to achieve high adherence to the treatment protocols, which is likely compatible with increased quality of care.

\section{Background}

Every year, over 30 million cases of malaria, pneumonia and diarrhoea in children go untreated in Uganda [1]. Under-five mortality from these three illnesses is 13,15

\footnotetext{
*Correspondence: pawor@musph.ac.ug

1 Department of Global Public Health and Primary Health Care, Centre for International Health, University of Bergen, PO Box 7800, 5020 Bergen, Norway

Full list of author information is available at the end of the article
}

and 9\%, respectively [2]. This large treatment gap and high under-five mortality can be reduced by ensuring high access to life saving preventive and curative services for these illnesses. However, access to health services in Uganda is variable, ranging from 7 to $100 \%$ in different parts of the country. Even where health facilities are available, low staffing, lack of necessary supplies and equipment and various health system challenges make access to basic care uncertain [1]. As a result of gaps within the 
public health sector, the private sector-dominated by drug shops in rural areas-has proliferated and is a major source of care for sick children [3]. More than half of sick children in Uganda seek care at the level of a drug shop when ill $[4,5]$. However, without any interventions at this level, the quality of care has been documented to be poor $[6,7]$.

Meanwhile, evidence shows that health workers, both public and private generally have low adherence to clinical protocols. For example, health workers in malaria endemic areas continue to prescribe artemisinin combination therapy to patients whose malaria diagnostic test result is negative, for various reasons including mistrust of test results [8-11]. Lower level health workers for example community health workers have been shown to follow clinical protocols more strictly $[12,13]$. However, the adherence of drug shop attendants to treatment protocols for malaria, pneumonia and diarrhoea is largely unknown. Given the role of drug shops in the care for children in Uganda and other low-income countries, it is necessary to understand adherence to treatment protocols in this group of health providers.

The objective of this study was to determine the level of adherence by drug shop attendants in eastern Uganda, to the integrated community case management (iCCM) of malaria, pneumonia and diarrhoea treatment guidelines.

\section{Methods}

This analysis was done with data collected during a quasi-experimental study determining the feasibility and effect on access and appropriateness of treatment with the introduction of the integrated community case management of malaria, pneumonia and diarrhoea intervention at drug shops. The study site, intervention, methods and results of the main study are published elsewhere [4]. For this paper, all drug shops $(\mathrm{N}=44)$ in the intervention area were included and all child visits $(\mathrm{N}=7,667)$ from October 2011-June 2012 to the participating drug shops were analysed.

\section{Data collection}

Drug shops maintained a standard iCCM register (see Additional file 1) where they recorded the children seen, their symptoms (fever, cough, fast/difficult breathing etc.), diagnostic test performed, the test results, treatment given and actions taken. For every child with fever, drug shop attendants were trained to perform a malaria rapid diagnostic test (RDT) and record the test result as positive or negative. Similarly for every child with cough and fast/difficult breathing, the drug shop attendants were trained to count the respiratory rate using the UNICEF respiratory rate (RR) counter and record the findings. Based on the standard iCCM sick-child-job-aid provided (see Additional files 2, 3), this child was then categorized as having pneumonia or not, taking into consideration the age and respiratory rate. Children with diarrhoea were also recorded in the register. After assessment, appropriate treatment was then given to the children.

\section{Quality control of diagnostic testing and treatment}

Quality control was done during the main study through support supervision. Both the local district drug inspector and a study nurse supervised the drug shops separately. After the iCCM training, a full time study nurse was employed to provide support supervision to the drug shop attendants. Initial support supervision was weekly at each drug shop and this was later reduced to monthly as less support became necessary. During the supervision visits, the study nurse observed the drug shop attendants manage children from the initial diagnosis of malaria, pneumonia or diarrhoea with utilization of the respective diagnostic test (RDT or RR counter), to the provision of appropriate treatment. The study nurse then discussed with the drug shop attendant the positive aspects and weaknesses observed during the management of the child. Continuous training was also provided to the drug shop attendant as necessary. More details of the support supervision process and results from some of the direct observation exercises have been previously published [4].

\section{Sample size}

To determine prevalence of adherence to clinical protocol from the registers, we used all records of children seen at the drug shops during the entire study period (8 months). The total sample size was 7,667 child visits to the drug shops.

\section{Analysis}

Data was double-entered in epi data [14] and was analysed using STATA, version 12 [15]. During the analysis, the symptoms and test results recorded in the iCCM registers were utilized, and every child was re-classified according to the iCCM algorithm. For children recorded as having fever, the analysis checked that an RDT result was recorded and whether appropriate treatment was given. For children recorded as having cough and fast/ difficult breathing, the analysis checked that a respiratory rate was recorded, then this respiratory rate was reclassified as fast or not (thus presence or absence of 'pneumonia'), based on the age of the child, as required by the guidelines. The appropriateness of treatment provided for pneumonia was assessed. For all children recorded as having diarrhoea, the analysis checked whether the recorded treatment was appropriate. Finally, the quality of integrated management of several concurrent 
symptoms was assessed. The proportion of children receiving a single, double, or triple illness classification was determined, and then the proportion of these children correctly assessed and treated was calculated. Confidence intervals around the estimates are provided.

\section{Ethics, consent and approvals}

Ethical approval was obtained from Uganda National Council of Science and Technology (\# HS 1184), the Uganda National Drug Authority (\# 0456/ID/NDA) and Makerere University School of Public Health (\# 166). Written consent was obtained from the drug sellers who participated in this study.

\section{Results}

A total of 7,667 children were seen at the drug shops during the eight months study period, from October 2011-May 2012. Of all recruited drug shops $(\mathrm{N}=44)$, two dropped out of the study in the initial months and another two were closed permanently, leaving 40 participating drug shops for the duration of the study. While half $(22 / 40)$ of the drug shops saw almost $90 \%$ of the children, just five drug shops saw nearly half of all the children. Table 1 shows the total number of children seen every month. Over the first 3 months the percentage of children seen at the drug shops was constant at about $11 \%$ of the total number of children seen. Over the remaining months of the study, there was an increase in the number of children seen $(\mathrm{p} \leq 0.001)$.

The children seen at the drug shops had a mean age of 21 months (range $0-84$ ) and $47 \%$ were 12 months and below, (Table 2). Eighty percent $(6,140 / 7,667)$ of all the children were recorded as having fever. Of these, 5986 (98\%) were tested for malaria with an RDT and the overall RDT positivity rate was 78\% (95\% CI 77-79). Further, 94\% of all children with a positive RDT test received the recommended malaria treatment, ACT.

Table 1 Total number of children seen at drug shops by month of study

\begin{tabular}{lcll}
\hline Month & Frequency & Percent & Test for trend \\
\hline October 2011 & 858 & 11.2 & $P \leq 0.001$ \\
November & 900 & 11.7 & \\
December & 883 & 11.5 & \\
January 2012 & 784 & 10.2 & \\
February & 950 & 12.4 & \\
March & 1,291 & 16.9 & \\
April & 850 & 11.1 & \\
May & 1,151 & 15.0 & \\
Total & 7,667 & 100 & \\
\hline
\end{tabular}

In this study, 5,203 (67.9\%) were assessed for fast breathing and $45 \%(3,437 / 7,667)$ were classified as having 'pneumonia' according to the MoH/WHO ICCM guidelines. The mean respiratory rate recorded was 48 breaths per minute (standard deviation 11.9). Of the children classified as having pneumonia, 91\% received the recommended drug, amoxicillin. Thirty-one percent $(2,335 / 7,667)$ of all the children seen at the shops had diarrhoea and $89 \%$ of these were recorded as having received the recommended treatment, ORS/zinc. Further, a total of $5 \%$ of children in the sample were classified as severely ill (having a danger sign) and the majority of these children were reported to have been referred to the health centre.

The proportion of children in the sample who were classified with both malaria and pneumonia was $31 \%$ and most of these $(93 \%)$ were recorded as having received both ACT for malaria and amoxicillin for pneumoniaTable 3. Meanwhile, 9\% of all the children seen at drug shops were found to have all three illnesses (malaria, pneumonia and diarrhoea) and the majority was treated appropriately.

\section{Discussion}

This analysis of treatment registers shows high adherence to the iCCM guidelines by drug shop attendants. Over $90 \%$ of all children with pneumonia-related symptoms, fever or diarrhoea were appropriately assessed, classified and treated according to guidelines. This is likely to represent an enormous step up in quality of care as illustrated by our previous paper where exit interviews and household surveys were used to assess appropriateness of treatment of children at the drug shops. That study found 3-13 times better management of children at drug shops with the introduction of iCCM [4]. Beside these two studies, there is limited additional evidence on the utilizing the iCCM strategy at the level of drug shops as studies at drug shops in low income countries have focused on single diseases especially malaria, at the expense of other febrile illness [16].

Similarly high levels of adherence to malaria RDT results by community health workers (97\%) have been demonstrated in Uganda and other African countries [12, $13,17]$. However, in two of these studies, the classification and treatment of pneumonia by CHWs was demonstrated to be poor with for example, only $40 \%$ of children with pneumonia symptoms being prescribed an antibiotic [12]. One child in three (31\%) received treatment for both malaria and pneumonia. While this confirms earlier findings by Källander et al. of a $30 \%$ symptom overlap [18], it also means that there was little further reduction in drug use from inclusion of RDTs compared to presumptive management. However, this was a high malaria 
Table 2 Classification and treatment of children attending drug shops

\begin{tabular}{|c|c|c|}
\hline Characteristic & Proportion & 95\% Confidence Intervals \\
\hline \multicolumn{3}{|l|}{ Child age } \\
\hline Up to 1 year & $3,596(46.9 \%)$ & \\
\hline Greater than 1 year & $4,071(53.1 \%)$ & \\
\hline Child sex-male & $3,732(48.8 \%)$ & \\
\hline \multicolumn{3}{|l|}{ Diarrhoea } \\
\hline Proportion with diarrhoea & $2,335 / 7,667(30.5 \%)$ & $29.4-31.5$ \\
\hline Received ORS packet & $2,068 / 2,335(88.6 \%)$ & $87.3-89.9$ \\
\hline \multicolumn{3}{|l|}{ Fever } \\
\hline Proportion with fever & $6,140 / 7,667(80.1 \%)$ & $79.2-81.0$ \\
\hline Fever and received Rapid Diagnostic test (RDT) & $5,986 / 6,140(97.5 \%)$ & \\
\hline Fever and RDT positive & $5,096 / 6,140(83.0 \%)$ & $82.1-83.9$ \\
\hline Received an RDT (ALL RDT tests done) & $6,801 / 7,667(88.7 \%)$ & \\
\hline RDT positive (overall RDT positivity) & $5,307 / 6,801(78.0 \%)$ & $77.0-79.0$ \\
\hline RDT positive and got ACT & $4,961 / 5,307(93.5 \%)$ & $92.8-94.1$ \\
\hline Any $A C T$ dispensed & $5,218 / 7,667(68.1 \%)$ & $67.0-69.1$ \\
\hline \multicolumn{3}{|l|}{ Pneumonia } \\
\hline Respiratory rate counted (all RRs counted) & $5,203 / 7,667(67.9 \%)$ & \\
\hline Proportion with pneumonia & $3,437 / 7,667(44.8 \%)$ & \\
\hline Pneumonia and amoxicillin dispensed & $3,126 / 3,437(91.0 \%)$ & \\
\hline Any amoxicillin dispensed & $3364 / 7,667$ (43.9\%) & $42.8-45.0$ \\
\hline \multicolumn{3}{|l|}{ Danger signs } \\
\hline Proportion with any danger sign & $381 / 7,667(5.0 \%)$ & \\
\hline Danger sign and referred & $309 / 381(81.1 \%)$ & \\
\hline \multicolumn{3}{|l|}{ Outcome } \\
\hline Referred & $477 / 7,667(6.2 \%)$ & $5.7-6.8$ \\
\hline Adverse drug reaction & $2 / 7,667$ & \\
\hline Died & $2 / 7,667$ & \\
\hline
\end{tabular}

Table 3 Management of double or triple illnesses in children, at drug shops

\begin{tabular}{lc}
\hline Both malaria and pneumonia & \\
Children with both malaria and pneumonia & $2,387 / 7,667(31.1 \%)$ \\
Received ACT & $2,237 / 2,387(93.7 \%)$ \\
Received Amoxicillin & $2,211 / 2,387(92.6 \%)$ \\
Malaria, pneumonia and diarrhoea & \\
Children with all 3 illnesses & $664 / 7,667(8.7 \%)$ \\
Received ACT & $624 / 664(94.0 \%)$ \\
Received Amoxicillin & $607 / 664(91.4 \%)$ \\
Received ORS/zinc & $580 / 664(87.3 \%)$ \\
\hline
\end{tabular}

transmission area with estimated parasite prevalence of $60 \%$ in school-age children $[19,20]$ and the RDT positivity rate at the drug shops from this study was $77 \%$. Limited penetration of malaria bed nets in this high malaria transmission area is likely to explain the high RDT positive rate, which will further have been augmented by the inherent problem of false positivity with malaria RDTdue to persistence of Plasmodium falciparum histidinerich protein 2 (PfHRP2) antigens four up to 1 month in the blood stream, following elimination of parasites [21, 22]. In comparison, Nankabirwa et al. reported 51\% malaria parasite prevalence using the gold-standard, microscopy, in children 0-59 months at public health facilities in regions with moderate-high malaria transmission in Uganda [23].

The high malaria positivity rate and the $45 \%$ of children whose respiratory rate was found high after assessment, meant that the drug shops could have continued, and perhaps even increased, the number of drugs sold per child, which coupled with the $25 \%$ increased utilization of these drug shops [4] likely increased their total financial turnover. Exactly how this affected total profits is not clear since we do not know profit margins of drugs earlier sold, although it was noted anecdotally that shopkeepers were almost uniformly happy saying "this has increased 
my business". However, how drug shop attendants would react in lower malaria endemic settings where a lower proportion of RDTs were positive and an increasing proportion of children are supposed to be treated with lower-profit-margin paracetamol is not clear, but needs to be subject to further study.

While we followed up 40 drug shops for the entire study period, care seeking tended to concentrate around particular drug shops. Five drug shops saw nearly half of all the children, during the entire study period. While this was unexpected, it is logical that parents may prefer certain characteristics of health workers/drug shop attendants and thus choose to take their children to these health workers. This concentration of care seeking has the potential to allow closer supervision, and also gaining more experience.

The discourse around governance of the private sector in pluralistic health systems emphasizes balancing both regulation and incentives $[24,25]$. Regulation alone may be insufficient as state regulation is weak and the processes for self-regulation are lacking. Incentives available to drug sellers for example through this iCCM intervention can improve quality of care, but there are limits to positive incentives alone. Community awareness to enable demand for quality service is also very important. This will likely need to involve aspects of asking for, and respecting test results, and an acceptance of treatment of children not with anti-malarials or antibiotics, but with for example paracetamol only if they have no malaria and no pneumonia.

This study has two important limitations that should be considered. First, the analysis is based on data from drug shop registers, which was not validated. However, this data was collected during a prospective experimental study and these results are very similar to results from the main study which used different data collection techniques like exit interviews and household surveys [4]. Secondly, the case definition of "pneumonia" in this study is not for confirmed pneumonia diagnosis, but for the iCCM classifications of 'pneumonia'-cough with fast/difficult breathing. This classification tends to result in more children being classified as having "pneumonia" than in reality.

\section{Conclusion}

This paper shows that with the introduction of the iCCM intervention at drug shops in Eastern Uganda, it was possible to achieve high adherence to the treatment protocols, which is likely compatible with increased quality of care.

\section{Additional files}

Additional file 1: The ICCM register: This is a copy of the standard book, where drug shop attendants (and community health workers) record all children seen with symptoms of malaria, pneumonia or diarrhoea.

Additional file 2: The sick child job aid 1. This is a visual aid that contains the iCCM treatment algorithm. It is printed in A3 size and displayed in the drug shop for easy reference when managing children.

Additional file 3: The sick child job aid 2. This is a visual aid that contains the iCCM treatment algorithm. It is printed in A3 size and displayed in the drug shop for easy reference when managing children.

\section{Authors' contributions}

PA, HW and SP conceptualized and designed the study; PA analysed the data and drafted the manuscript which all authors edited and approved. All authors read and approved the final manuscript.

\section{Author details}

${ }^{1}$ Department of Global Public Health and Primary Health Care, Centre for International Health, University of Bergen, PO Box 7800, 5020 Bergen, Norway. ${ }^{2}$ Department of Community Health and Behavioural Sciences, School of Public Health, College of Health Sciences, Makerere University, PO Box 7072, Kampala, Uganda. ${ }^{3}$ Global Health, Karolinska Institutet, Stockholm, Sweden.

${ }^{4}$ International Maternal and Child Health Unit, Uppsala University, Uppsala, Sweden.

\section{Acknowledgements}

We are grateful to the Einhorn Family Foundation, Sweden, Swedish Science Council and Medicines for Malaria Venture, Switzerland, for funding this study.

\section{Compliance with ethical guidelines}

\section{Competing interests}

The authors declare that they have no competing interest.

Received: 9 March 2015 Accepted: 7 July 2015

Published online: 16 July 2015

\section{References}

1. $\mathrm{MoH}$ (2010) Integrated community case management of childhood malaria, pneumonia and diarrhoea: implementation guide. Ministry of Health CHD, Kampala

2. Uganda Health Profile (http://www.who.int/gho/countries/uga. pdf?ua=1). Accessed 1 Dec 2014

3. Tougher S, Ye Y, Amuasi JH, Kourgueni IA, Thomson R, Goodman C et al (2012) Effect of the Affordable Medicines Facility-malaria (AMFm) on the availability, price, and market share of quality-assured artemisinin-based combination therapies in seven countries: a before-and-after analysis of outlet survey data. Lancet 380:1916-1926

4. Awor P, Wamani H, Tylleskar T, Jagoe G, Peterson S (2014) Increased access to care and appropriateness of treatment at private sector drug shops with integrated management of malaria, pneumonia and diarrhoea: a quasi-experimental study in Uganda. PLoS One 9:e115440

5. Rutebemberwa E, Pariyo G, Peterson S, Tomson G, Källander K (2009) Utilization of public or private health care providers by febrile children after user fee removal in Uganda. Malar J 8:45

6. Wafula FN, Miriti EM, Goodman CA (2012) Examining characteristics, knowledge and regulatory practices of specialized drug shops in subSaharan Africa: a systematic review of the literature. BMC Health Serv Res $12: 223$ 
7. Awor P, Wamani H, Bwire G, Jagoe G, Peterson S (2012) Private sector drug shops in integrated community case management of malaria, pneumonia, and diarrhea in children in Uganda. Am J Trop Med Hyg 87:92-96

8. Chandler Cl, Whitty CJ, Ansah EK (2010) How can malaria rapid diagnostic tests achieve their potential? A qualitative study of a trial at health facilities in Ghana. Malar J 9:95

9. Kyabayinze J (2010) Use of RDTs to improve malaria diagnosis and fever case management at primary health care facilities in Uganda. In: World Malaria Day 3rd Technical and Scientific Symposium. Protea Hotel, Kampala, Uganda: Ministry of Health, Uganda

10. Ansah EK, Narh-Bana S, Epokor M, Akanpigbiam S, Quartey AA, Gyapong $J$, et al. (2010) Rapid testing for malaria in settings where microscopy is available and peripheral clinics where only presumptive treatment is available: a randomised controlled trial in Ghana. Bmj 340:c930

11. Bastiaens GJ, Bousema T, Leslie T (2014) Scale-up of malaria rapid diagnostic tests and artemisinin-based combination therapy: challenges and perspectives in sub-Saharan Africa. PLoS Med 11:e1001590

12. Mukanga D, Babirye R, Peterson S, Pariyo GW, Ojiambo G, Tibenderana JK et al (2011) Can lay community health workers be trained to use diagnostics to distinguish and treat malaria and pneumonia in children? Lessons from rural Uganda. Trop Med Int Health 16:1234-1242

13. Kalyango JN, Rutebemberwa E, Alfven T, Ssali S, Peterson S, Karamagi C (2012) Performance of community health workers under integrated community case management of childhood illnesses in eastern Uganda. Malar J 11:282

14. EpiData software (http://www.epidata.dk). Accessed 1 Dec 2014

15. STATA data anlysis and statistical software (http://www.stata.com) Accessed 1 Dec 2014

16. Awor P, Miller J, Peterson S (2014) Systematic literature review of integrated community case management and the private sector in Africa: relevant experiences and potential next steps. J Glob Health 4:020414
17. Mukanga D, Tiono AB, Anyorigiya T, Källander K, Konate AT, Oduro AR et al (2012) Integrated community case management of fever in children under five using rapid diagnostic tests and respiratory rate counting: a multi-country cluster randomized trial. Am J Trop Med Hyg 87:21-29

18. Kallander K, Nsungwa-Sabiiti J, Peterson S (2004) Symptom overlap for malaria and pneumonia-policy implications for home management strategies. Acta Trop 90:211-214

19. CAPSS (2011) Closing the affordability gap: Ensuring affordable and effective malaria treatment for children in rural Uganda. Subsidized ACTs in the private sector, CAPSS MOH-MMV Pilot, 2011. http://Www.mmv.org/ sites/default/files/uploads/docs/access/Access_projects/Closing_the_ Affordability_Gap.pdf

20. UBOS (2015) Uganda Malaria Incator Survey 2014-2015: key indicators. Uganda Bureau of statistics (UBOS)and ICF international, Kampala, Uganda and Rockville

21. Mouatcho JC, Goldring JP (2013) Malaria rapid diagnostic tests: challenges and prospects. J Med Microbiol 62:1491-1505

22. Moody A (2002) Rapid diagnostic tests for malaria parasites. Clin Microbiol Rev 15:66-78

23. Nankabirwa J, Zurovac D, Njogu JN, Rwakimari JB, Counihan H, Snow RW et al (2009) Malaria misdiagnosis in Uganda-implications for policy change. Malar J 8:66

24. Bennett S, Bloom G, Knezovich J, Peters DH (2014) The future of health markets. Global Health 10:51

25. Bloom G, Henson S, Peters DH (2014) Innovation in regulation of rapidly changing health markets. Global Health 10:53

\section{Submit your next manuscript to BioMed Central and take full advantage of:}

- Convenient online submission

- Thorough peer review

- No space constraints or color figure charges

- Immediate publication on acceptance

- Inclusion in PubMed, CAS, Scopus and Google Scholar

- Research which is freely available for redistribution

Submit your manuscript at

www.biomedcentral.com/submit

C BioMed Central 